\title{
Neutropenie-Prophylaxe: G-CSF-Präparate im Vergleich
}

G-CSF („granulocyte-colony stimulating factor") beugt schweren Neutropenien unter Chemotherapie vor. Was ist effektiver: Filgrastim mit täglicher Gabe oder die Einmalgabe von Pegfilgrastim?

Z ur Prophylaxe der Neutropenie unter Chemotherapie stehen Pegfilgrastim (pegyliertes G-CSF) zur einmaligen Applikation pro Zyklus oder Filgrastim für die tägliche subkutane Gabe an den Tagen 2-7 zur Auswahl. Head-to-Head-Vergleichsstudien beider Optionen sind nicht selten interessengesteuert, für einen objektiveren Vergleich wurde daher retrospektiv auf Daten von Studien zurückgegriffen, in denen ursprünglich andere Zielparameter als der direkte Vergleich vorgesehen waren. Bei den 1.058 Brustkrebspatientinnen waren die Endpunkte febrile Neutropenie (FN), Grad-3/4-Neutropenie sowie CT-Regime-Änderungen

\begin{tabular}{|l|l|l|l|}
\hline \begin{tabular}{l} 
Tab.1: Vergleich einiger Variablen unter den beiden Substanzen \\
\hline
\end{tabular} Filgrastim $(\mathbf{n}=\mathbf{5 2 9})$ & Pegfilgrastim $(\mathbf{n = 5 2 9 )}$ & p-Wert \\
\hline Febrile Neutropenie & $3,4 \%$ & $4,3 \%$ & 0,500 \\
\hline Schwere Neutropenie & $32,3 \%$ & $10,4 \%$ & $<0,001$ \\
\hline Therapieverzögerung (> 2 Tage) & $42,0 \%$ & $27,6 \%$ & $<0,001$ \\
\hline Dosisreduktion & $18,5 \%$ & $10,8 \%$ & $<0,001$
\end{tabular}

erfasst worden. Von 18 Episoden einer FN $(3,4 \%)$ in der Filgrastim- und $23(4,3 \%)$ in der Pegfilgrastim-Gruppe $(\mathrm{p}=0,50)$ traten über die Hälfte in den ersten $4 \mathrm{Zy}$ klen auf. Die Patientinnen hatten unter Filgrastim ein fast 3-fach höheres Risiko für eine schwere Neutropenie (Tab. 1) als unter Pegfilgrastim und die Chemotherapie-Dosis musste signifikant häufiger reduziert werden (18,5 vs. $10,8 \%$; $\mathrm{p}<0,001)$. Der Anteil der Patientinnen, die ihr Regime wie geplant erhielten, war unter Filgrastrim signifikant geringer (58, 0 vs. $72,4 \%$; $\mathrm{p}<0,001)$.

Fazit: Pegfilgrastim beugt im Vergleich zu Filgrastim effizienter schweren, nichtfebrilen Neutropenien vor und reduziert CT-Behandlungsverzögerungen und Dosisreduktionen. Barbara Kreutzkamp

Kourlaba $\mathrm{G}$ et al. Comparison of filgrastrim and pegfilgrastrim to prevent neutropenia and maintain dose intensity of adjuvant chemotherapy in patients with breast cancer. Support Care Cancer. 2015;23(7):2045-51.

\section{Harnsäureblocker gegen Tumorlysesyndrom}

\section{Der Xanthinoxidase-Blocker Febuxostat ist zur Behandlung der Gicht zuge- lassen. Womöglich könnte er daher auch dem vor allem bei hämatologisch- onkologischen Erkrankungen gefürchteten Tumorlysesyndrom vorbeugen. Hier gehört der Serum-Harnsäurespiegel zu den Warnsymptomen.}

D as Tumorlysesyndrom (TLS) tritt besonders häufig bei hämatologischen Malignomen auf. Dabei kommt es durch den Zerfall vieler Tumorzellen zu metabolischen Entgleisungen bis hin zu Nierenversagen und kardialen Arrhythmien, neurologischen Komplikationen sowie plötzlichen Todesfällen. Warnsymptom ist eine starke Erhöhung des Serum-Harnsäurespiegels. Die aktuelle Richtlinie empfiehlt die Gichtmittel Allopurinol und Rasburicase zur Vorbeugung. Bei der Behandlung einer durch die Gicht bedingten Hyperurikämie scheint der neue Xanthinoxidase-Inhibitor Febuxostat aber zumindest Allopurinol überlegen zu sein. Ob er sich auch besser zur Prophylaxe des TLS eignet, sollte in der internationalen, multizentrischen Phase-III-Studie FLORENCE untersucht werden.

Dafür wurden Patienten mit hämatologischen Malignomen und mittlerem bis hohem Risiko für ein TLS randomisiert. Sie erhielten ab zwei Tage vor der Induktionstherapie für 7-9 Tage doppelblind entweder $120 \mathrm{mg}$ Febuxostat oder Allopurinol. Primäre Endpunkte waren die Harnsäurekonzentration im Serum (AUC) sowie SerumkreatininVeränderungen, sekundäre Endpunkte Ansprechrate, Inzidenz des TLS und Sicherheit der Therapie.

346 Patienten wurden randomisiert. Von ihnen hatten $82,1 \%$ ein mittleres Risiko für ein TLS. Die mittlere AUC der
Harnsäure lag bei 514,0 gegenüber 708,0 zugunsten von Febuxostat $(\mathrm{p}<0,0001)$. Die mittleren Serumkreatinin-Veränderungen betrugen unter Febuxostat $-0,83$ und unter Allopurinol -4,92 ( $\mathrm{p}=0,0903)$. Zwischen den sekundären Endpunkten ergaben sich keine Unterschiede. Nebenwirkungen zeigten sich bei $6,4 \%$ der Patienten in beiden Armen.

Fazit: In der bisher größten Studie zur Prävention des TLS bei Erwachsenen senkte Febuxostat die Harnsäurekonzentration signifikant besser als Allopurinol. Die Inzidenz des TLS war allerdings unter beiden Therapien gleich häufig. Die renale Funktion war in beiden Behandlungsarmen ebenso vergleichbar wie das Nebenwirkungsprofil.

Christina Berndt

Spina $M$ et al. FLORENCE: a randomized, double-blind, phase III pivotal study of febuxostat versus allopurinol for the prevention of tumor lysis syndrome (TLS) in patients with hematologic malignancies at intermediate to high TLS risk. Ann Oncol. 2015;26(10):2155-61. 